\title{
A simple model for neural computation with firing rates and firing correlations
}

\author{
Wolfgang Maass $\dagger$ \\ Institute for Theoretical Computer Science, Technische Universität Graz, Klosterwiesgasse 32/2, \\ A-8010 Graz, Austria \\ Received 17 October 1997, in final form 29 April 1998
}

\begin{abstract}
A simple extension of standard neural network models is introduced which provides a model for neural computations that involve both firing rates and firing correlations. Such an extension appears to be useful since it has been shown that firing correlations play a significant computational role in many biological neural systems. Standard neural network models are only suitable for describing neural computations in terms of firing rates.

The resulting extended neural network models are still relatively simple, so that their computational power can be analysed theoretically. We prove rigorous separation results, which show that the use of firing correlations in addition to firing rates can drastically increase the computational power of a neural network.

Furthermore, one of our separation results also throws new light on a question that involves just standard neural network models: we prove that the gap between the computational power of high-order and first-order neural nets is substantially larger than shown previously.
\end{abstract}

\section{Introduction}

A large number of results in experimental neurophysiology suggest that in addition to firing rates, correlations between firing times of neurons are also relevant for neural coding and computation (see e.g. deCharms and Merzenich 1996, Engel et al 1992, Eggermont 1990, Kreiter and Singer 1996, Krüger 1991, MacLeod and Laurent 1996, Sillito et al 1994, Singer 1995, Vaadia et al 1995). The standard neural network models, such as threshold circuits or sigmoidal neural nets (MLPs, i.e. multi-layer perceptrons), are not suitable for evaluating the potential capabilities and limitations of such neural systems, since they are tailored to modelling neural computation just in terms of firing rates.

There exist, of course, substantially more detailed mathematical models for biological neural systems, where one can study correlations between firing times, such as networks of integrate-and-fire neurons or 'spiking neurons' (see e.g. Bower and Beeman 1995, Gerstner 1995, Maass 1996, 1997a, Tuckwell 1988). However, these models have the disadvantage that they keep track of every single firing of each neuron in the system, which makes them less suitable for exploring analytically those types of neural computations where only the high-level statistical correlations of firing times are relevant for the neural computation.

We introduce in this article a simple extension of the familiar abstract neural network models (i.e. of threshold-circuits and sigmoidal neural nets) that allows us to model also

$\dagger$ E-mail: maass@igi.tu-graz.ac.at 
salient computational features of correlations between firing times in this simplified setting. Several models that reflect computational effects of firing correlations in a simplified setting have already previously been proposed (Milner 1974, von der Malsburg 1981, Eckhorn et al 1990, Abeles 1991, Lumer and Huberman 1992, Shastri and Ajjanagadde 1993, Phillips and Singer 1997). All these earlier models focus on special purpose computations, such as selective input enhancement, or 'binding' of different features of an object. One has not been able to prove separation results that distinguish the computational power of these models from that of standard neural network models. Since standard sigmoidal neural nets are 'universal approximators', it is clear that there exist sigmoidal neural nets that compute the same functions as the previously mentioned more complex models which also involve computational effects of firing correlations. Therefore it is impossible to demonstrate a computational advantage of firing correlations by showing that they allow the computation of certain functions that cannot be computed without firing correlations. Instead, the essential question is a quantitative one: for example, by how much larger would a standard neural network model have to be in order to compute the same function as some network that also models firing correlations. This question has not been answered for the earlier models. In contrast to these earlier approaches, we consider in the article not just special purpose computations, but we model the role of firing correlation within the context of a general computational model. Furthermore we prove rigorous quantitative results about the computational power of this augmented computational model in comparison with standard neural network models.

So far one has mainly tried to understand the use of firing correlations in sensory cortical systems in the context of symbolic concepts such as 'binding' of features. On the other hand, there exist many artificial systems that work quite well for 'real world' noisy pattern recognition tasks with very large numbers of diverse inputs, but all these systems are based on quite different approaches (see e.g. Weiss and Kulikowski 1991, Ripley 1996, Mel 1997). Hence it is conceivable that sensory neural systems in the cortex also involve complex computations involving firing correlation that cannot be adequately understood in psychological terms such as 'binding'. This argument raises the question of which other computational role could firing correlations possibly play for pattern recognition in complex sensory neural systems. The general purpose computational model introduced in this paper provides a framework for approaching this question.

An independent motivation for investigating the possible computational role of firing correlations arises from recent experiments with new electronic hardware. In the context of attempts to build reliable devices for signal processing and computation in the microwatt range, one has started to explore silicon implementations of networks of spiking neurons, or 'pulsed VLSI' (see e.g. Murray and Tarassenko 1994, Zaghoul et al 1994). Very recently (see van Schaik et al 1997) one has also started to explore the computational use of correlations among different pulse streams. However, the question of how correlations between pulse streams can best be used in a technological context remains wide open. The model introduced in this paper provides a framework where possible uses of pulse correlations in pulsed VLSI can be analysed and compared with other computational models and known mechanisms of biological neural systems.

In the next section we define our model for computations with firing rates and firing correlations. We explore the computational power of a single unit of this model in section 3. In section 4 we analyse the computational power of multi-layer versions of this model. Conclusions are given in section 5. 


\section{Definition of the model}

In the usual neural network models one reserves for each gate (or 'unit') $u$ of the network a formal variable $o(u)$ which denotes the output of $u$. In a biological interpretation this variable $o(u)$ models the current firing rate of a neuron $u$. We will now consider a new type of neural network model $\mathcal{N}$ where one has, in addition to the formal variables $o(u)$ for each gate $u$ of $\mathcal{N}$, a second type of formal variable $c(S)$ for various sets $S$ of gates in $\mathcal{N}$. In a biological interpretation the formal variable $c(S)$ models the current correlation in the firing times of neurons in this set $S$. A characteristic feature of this new type of variables is that no additional computational units are needed to compute their value. Instead, their values are determined through the collective activity of the gates according to our equation (2.2). In particular, a computation may involve many more variables $c(S)$ than there are gates in the network.

We assume that an arbitrary directed graph is given that describes the architecture of $\mathcal{N}$. Each gate $v$ of the network $\mathcal{N}$ receives both types of variables as input, i.e. in addition to the variables $o(u)$ for immediate predecessors $u$ it also receives the variables $c(S)$ for subsets $S$ of its set of immediate predecessors. The output $o(v)$ of $v$ depends on both types of inputs, reflecting the biological property that the firing rate of a neuron may depend both on the firing rates of its predecessors and on the firing correlations among subsets of its predecessors. Dually, in addition to the variable $o(v)$ the input of $v$ also contributes to the values of the variables $c(T)$ for sets $T$ with $v \in T$.

Thus, besides the 'real' variables $o(u)$, gates in $\mathcal{N}$ also handle a second type of 'imaginary' variables $c(S)$. Furthermore, the computational operation of a gate $v$ cannot be decomposed into its operation on 'real' variables and its operation on 'imaginary' variables, since both its 'real' output $o(v)$ and its 'imaginary' output $c(T)$ depend on both types of input variables. Hence the computational operation of such a gate $v$ is reminiscent of a complex function in mathematics (for example, $z \mapsto \mathrm{e}^{z}$, where the real and imaginary components of the output $\mathrm{e}^{z}$ for an input $z=x+\mathrm{i} y$ depend on both $x$ and $y$ ). Because of this loose analogy we refer to the new type of gates for neural networks that are investigated in this article as complex gates $\dagger$. In contrast, we will refer to traditional gates of neural network models as standard gates. A standard neural net is in our terminology a neural net consisting of standard gates.

The 'real' component $o(v)$ of the output of a complex gate $v$ is described by the equation

$$
o(v)=\sigma\left(\sum_{u \in U} \alpha_{v u} \cdot o(u)+\sum_{S \subseteq U} \alpha_{v S} \cdot c(S) \cdot \prod_{u \in S} o(u)+\alpha_{v}\right)
$$

where $U$ is the set of immediate predecessors of $v$ in the directed graph that describes the architecture of the network $\mathcal{N}$. The parameters $\alpha_{v u}, \alpha_{v s}$ and $\alpha_{v}$ may have arbitrary real values. We refer to parameters of the form $\alpha_{v u}$ and $\alpha_{v S}$ as weights, and to $\alpha_{v}$ as the bias of the complex gate $v$. The product $c(S) \cdot \prod_{u \in S} o(u)$ in (2.1) models the following effect: a firing correlation of the neurons in $S$ can increase the firing rate of $v$ if simultaneously all neurons in $S$ fire above their resting firing rate (see Bernander et al (1994) for detailed simulation results). The letter $\sigma$ in (2.1) denotes some arbitrary activation function $\sigma: \mathbf{R} \rightarrow \mathbf{R}$, that can be chosen as in traditional neural network models. If $\sigma$ is the Heaviside function (defined by $\sigma(y)=1$ if $y \geqslant 0$ and $\sigma(y)=0$ if $y<0$ ) we refer to such a gate as a complex threshold gate. If $\sigma$ is a smooth squashing function (such as the logistic sigmoid $\sigma(y)=1 /\left(1+\mathrm{e}^{-y}\right)$ ) we refer to the gate defined by (2.1) as a complex sigmoidal gate.

$\dagger$ One should be aware, however, that this analogy is quite imperfect. In particular, the computation of the 'imaginary' part $c(T)$ cannot be allocated to a particular complex gate; see equation (2.2) 
For any set $T$ of gates, the value of the variable $c(T)$ is determined by the equation

$$
c(T)=\sigma_{c}\left(\sum_{u \in U} \alpha_{T u} \cdot o(u)+\sum_{S \subseteq U} \alpha_{T S} \cdot c(S) \cdot \prod_{u \in S} o(u)+\alpha_{T}\right) .
$$

This equation is not relevant for the computational problems discussed in this section or section 3. Hence we defer its discussion to section 4, where we will look at multi-layer computations with complex gates.

Equations (2.1) and (2.2) define special cases of high-order sigmoidal gates, where the order refers to the degree of the polynomial in the variables $o(u)$ and $c(S)$ to which the activation function $\sigma$ is applied. Hence networks of complex sigmoidal gates are special cases of high-order sigmoidal networks, i.e. sigmoidal neural nets where the sigmoidal activation functions of the gates are applied to a polynomial of its inputs with degree $>1$. A number of different mechanisms have already been suggested through which such highorder sigmoidal gates might be implemented by biological neurons (a very good survey is given in Koch and Poggio (1992)). The model that is discussed in this article suggests firing correlations as yet another (and possibly somewhat unexpected) candidate for a mechanism through which at least some special types of high-order sigmoidal gates can in principle be implemented in networks of biological neurons. High-order gates have previously been discussed in various contexts in the neural network literature (see e.g. Durbin and Rumelhart 1989, Koch and Poggio 1992, Bruck and Smolenski 1992, Omlin and Giles 1996, Maass 1997b). It is well known that every digital or analogue function that can be computed or approximated by a high-order neural network can also be computed or approximated by a first-order neural network. Hence it is impossible to show that certain computations can be carried out with the help of high-order gates that are not possible with first-order gates. One can only demonstrate a computational advantage of high-order gates by analysing whether they can compute a function with a less complex network. This becomes technically very difficult, since one has to prove that no first-order network of similar complexity could compute the same function. Negative results of this type are very rare, due to a general lack of techniques for proving lower bounds in computational complexity theory. We introduce in this article a new method for showing rigorously that high-order neural networks can compute some concrete functions with a less complex network than first-order nets. This result and related literature will be discussed in section 3 before the general lower bound result is given in corollary 3.2 .

If one assumes that the firing rate $o(u)$ of each input neuron $u$ is proportional to the probability $p_{u}(I)$ that neuron $u$ fires during some fixed short time-window $I$ (say of length $2 \mathrm{~ms}$ ), and if the spike trains from a set $S$ of such neurons $u$ can be modelled by independent stochastic processes, then the probability that all neurons $u \in S$ fire during this time-window $I$ is given by $\prod_{u \in S} p_{u}(I)$. If the spike trains from these neurons $u \in S$ are not generated by independent stochastic processes, one can interpret the value of the variable $c(S)$ as

$$
\frac{\operatorname{Pr}[\text { all neurons } u \in S \text { fire during } I]}{\prod_{u \in S} p_{u}(I)} .
$$

Then the term $c(S) \cdot \prod_{u \in S} o(u)$ in equation (2.1) is equal to $\operatorname{Pr}[$ all neurons $u \in$ $S$ fire during $I$ ]. The actual impact that a larger than 'usual' (i.e. larger than $\prod_{u \in S} p_{u}(I)$ ) probability of synchronous firing of input neurons $u \in S$ may have on the firing rate of $v$ is scaled in equation (2.1) by some generalized 'weight' $\alpha_{v S}$.

In a biological interpretation the value $\alpha_{v S}$ depends on the locations of the synapses between neurons $u \in S$ and $v$ on the axonal tree of the presynaptic neurons $u$ and on the 
dendritic tree or soma of the postsynaptic neuron $v$. In addition it depends on the biochemical structure of these synapses, and on the distribution of voltage-dependent channels on the dendritic tree of $v$. For example, if the transmission delays between the neurons $u \in S$ and their synapses on $v$ all have roughly the same values, if all neurons in $u$ have synapses close together on the dendritic tree of $v$, and if there exists an accumulation of voltage-dependent channels in a nearby branching point on the way to the soma of $v$ (so that a 'dendritic spike' can be generated at such a 'hot spot'), then an increase in the firing correlation of the neurons in $S$ is likely to have a significant impact on the firing rate of neuron $v$, and $\alpha_{v S}$ should be given a relatively large value. In a similar way the parameters $\alpha_{T u}$ and $\alpha_{T S}$ in equation (2.2) also provide a possibility to reflect salient computational aspects of the specific geometrical and biochemical structure of biological neurons (see the discussion at the beginning of section 4). We refer to Mel (1994) for further information on biological details of dendritic integration.

Our simple model gives rise to a number of interesting new research questions regarding computations with biological neurons. One question is whether all features of concrete biological neurons that are relevant for their computations in terms of firing rates and firing correlations can be captured through suitable choices of the parameters involved in equations (2.1) and (2.2). In addition there arise the questions of how many of these parameters can be chosen independently by a biological neuron, and which learning algorithms govern the determination of these parameters in biological neural systems.

An interpretation of $c(S)$ according to (2.3) suggests that $c(S)$ may assume arbitrary real values in the range $[0, \infty]$. However, similarly to the variables $o(u)$ in standard neural network models, we allow these new variables $c(S)$ to be interpreted in a more abstract way. Their actual range depends in our model on the specific activation functions $\sigma_{c}$ that are employed.

\section{The computational power of a complex threshold gate}

In this section we exhibit concrete computational problems for which one can prove that they can be computed more efficiently by a complex threshold gate. More precisely, we identify a class of boolean functions $F$ that can be computed by a single complex threshold gate, whereas any feedforward threshold circuit or sigmoidal neural net computing the same function $F$ needs to have many gates. We will discuss after theorem 3.1 four concrete instances of such functions $F$ which are assumed to be related to fundamental computational tasks of biological neural systems.

Let $\mathcal{C}$ be some arbitrary class of non-empty subsets of $\left\{u_{1}, \ldots, u_{n}\right\}$. We consider the boolean function $F_{\mathcal{C}}:\{0,1\}^{m} \rightarrow\{0,1\}$ for $m:=n+|\mathcal{C}| . F_{\mathcal{C}}$ gives for input vectors $\left\langle x_{i}\right\rangle_{i=1, \ldots, n}{ }^{\cap}\left\langle x_{S}\right\rangle_{S \in \mathcal{C}}$ from $\{0,1\}^{m}$ the output 1 if and only if $x_{S} \cdot \prod_{u_{i} \in S} x_{i}=1$ for some $S \in \mathcal{C}$. The operation ${ }^{\cap}$ denotes the concatenation of vectors.

Theorem 3.1. The function $F_{\mathcal{C}}:\{0,1\}^{m} \rightarrow\{0,1\}$ can be computed by a single complex threshold gate.

Let $\tilde{\mathcal{C}} \subseteq \mathcal{C}$ be an arbitrary collection of sets in $\mathcal{C}$ so that no $S, S^{\prime} \in \tilde{\mathcal{C}}$ exist with $S \varsubsetneqq S^{\prime}$. Then any standard feedforward threshold circuit computing $F_{\mathcal{C}}$ needs to have at least $|\tilde{\mathcal{C}}| / \log (|\tilde{\mathcal{C}}|+1)$ gates, and any standard feedforward sigmoidal neural net with piecewise rational activation functions needs to have $\Omega\left(|\tilde{\mathcal{C}}|^{1 / 2}\right)$ gates. For standard sigmoidal neural nets whose activation function involves exponentiation one gets a lower bound of $\Omega\left(|\tilde{\mathcal{C}}|^{1 / 4}\right)$ for the required number of gates. 
Proof. The computation of $F_{\mathcal{C}}$ by a single complex threshold gate $v$ is straightforward. We assume that $v$ receives the input variables $x_{i}$ in the form of activations $o\left(u_{i}\right)=x_{i}$ of its input neurons $u_{1}, \ldots, u_{n}$, and the input variables $x_{S}$ in the form of correlations $c(S)$ of the corresponding set $S$ of input variables. One sets $\alpha_{v u_{i}}=0$ for $i=1, \ldots, n, \alpha_{v S}=1$ if $S \in \mathcal{C}$ and $\alpha_{v S}=0$ if $S \notin \mathcal{C}$, and $\alpha_{v}=-\frac{1}{2}$. With this parameter assignment the complex threshold gate $v$ computes the given function $F_{\mathcal{C}}$.

Let $\mathcal{N}$ be some arbitrary standard feedforward threshold circuit or sigmoidal neural net with $m$ binary input variables $\left\langle x_{i}\right\rangle_{i=1, \ldots, n} \cap\left\langle x_{S}\right\rangle_{S \in \mathcal{C}}$ that computes $F_{\mathcal{C}}$. In the case of a sigmoidal neural net we assume that the real-valued output of its output gate is rounded to 0 or 1 to yield a boolean-valued output.

Assume that $\ell$ gates of $\mathcal{N}$ have a direct edge from at least one of the input nodes for the input variables $x_{S}, S \in \mathcal{C}$. Obviously $\ell$ is not larger than the total number of gates (i.e. computation nodes) in $\mathcal{N}$.

Let $\tilde{\mathcal{N}}$ be a variation of $\mathcal{N}$ whose only input variables are $x_{1}, \ldots, x_{n}$, and where the biases of those $\ell$ gates in $\mathcal{N}$ that have a direct edge from one of the input nodes for the input variables $\left\{x_{S}: S \in \mathcal{C}\right\}$ are the only 'programmable parameters' of $\tilde{\mathcal{N}}$. All other weights and biases in $\tilde{\mathcal{N}}$ have the same values as in $\mathcal{N}$. The input nodes for the variables $x_{S}, S \in \mathcal{C}$, are no longer present in $\tilde{\mathcal{N}}$. For arbitrary $\mathbf{c} \in \mathbf{R}^{\ell}$ we write $\tilde{\mathcal{N}}^{\mathbf{c}}$ for the network that results if the values in $\mathbf{c}$ are assigned to the $\ell$ programmable parameters of $\tilde{\mathcal{N}}$.

Consider the set

$$
D:=\left\{\left\langle a_{1}, \ldots, a_{n}\right\rangle \in\{0,1\}^{n}: \exists S\left(S \in \tilde{\mathcal{C}} \wedge S=\left\{u_{i}: a_{i}=1\right\}\right) .\right.
$$

This is the set of all inputs for $\tilde{\mathcal{N}}$ that encode the 'characteristic function' of some set $S \in \tilde{\mathcal{C}}$.

We show that $\tilde{\mathcal{N}}$ (or more precisely the class of all boolean functions from $\{0,1\}^{n}$ into $\{0,1\}$ that are computed by $\tilde{\mathcal{N}}^{\mathbf{c}}$ for different $\mathbf{c} \in \mathbf{R}^{\ell}$ ) 'shatters' the set $D$. In other words, we show that for every $A \subseteq D$ there exists some $\mathbf{c} \in \mathbf{R}^{\ell}$ such that $\tilde{\mathcal{N}}^{\mathbf{c}}$ outputs the value 1 for an input $\mathbf{a} \in D$ if and only if $\mathbf{a} \in A$.

Thus, we fix some arbitrary $A \subseteq D$. Let $\mathbf{b}_{A} \in\{0,1\}^{|\mathcal{C}|}$ be a corresponding assignment to the input variables $\left\{x_{S}: S \in \mathcal{C}\right\}$ of $\mathcal{N}$ which assigns to $x_{S}$ the value 1 if and only if $S=\left\{u_{i}: a_{i}=1\right\}$ for some $\left\langle a_{1}, \ldots, a_{n}\right\rangle \in A$. The fixed assignment of $\mathbf{b}_{A}$ to the input variables $\left\langle x_{S}\right\rangle_{S \in \mathcal{C}}$ of $\mathcal{N}$ can be modelled in $\tilde{\mathcal{N}}$ by a corresponding assignment $\mathbf{c}_{A}$ to the $\ell$ programmable parameters of $\tilde{\mathcal{N}}$ : i.e. the contribution of these input variables $x_{S}$ to the weighted sums of the those $\ell$ gates that have edges from input nodes for these input variables $x_{S}$. Then $\tilde{\mathcal{N}}^{\mathbf{c}_{A}}$ computes the same function from $\{0,1\}^{n}$ into $\{0,1\}$ as the net $\mathcal{N}$ with the fixed assignment $\mathbf{b}_{A}$ to its input variables $\left\langle x_{S}\right\rangle_{S \in \mathcal{C}}$. Since $\mathcal{N}$ computes $F_{\mathcal{C}}$ and since no set in $\tilde{\mathcal{C}}$ is contained in another set in $\tilde{\mathcal{C}}$, it outputs by definition of $\mathbf{b}_{A}$ the value 1 for input $\left\langle a_{1}, \ldots, a_{n}, \mathbf{b}_{A}\right\rangle$ from $\{0,1\}^{m}$ if and only if $\left\langle a_{1}, \ldots, a_{n}\right\rangle \in A$. Hence $\tilde{\mathcal{N}}^{\mathbf{c}}$ with input $\left\langle a_{1}, \ldots, a_{n}\right\rangle \in D$ outputs 1 if and only if $\left\langle a_{1}, \ldots, a_{n}\right\rangle \in A$.

The preceding argument shows that the neural net $\tilde{\mathcal{N}}$ with its $\ell$ programmable parameters shatters the set $D$ of size $|\tilde{\mathcal{C}}|$. Hence the VC-dimension of $\tilde{\mathcal{N}}$ is at least $|\tilde{\mathcal{C}}|$. Since $\tilde{\mathcal{N}}$ has $\ell$ programmable parameters, any upper bound $B(\ell)$ for its VC-dimension induces for $\ell$ a lower bound of the form

$$
\text { least } \ell \text { such that } B(\ell) \geqslant|\tilde{\mathcal{C}}| \text {. }
$$

By the definition of $\ell$ this argument yields the same lower bound for the number of gates in $\mathcal{N}$. We would like to point out that such an argument has first been used (in a different context) by Koiran (1996).

In the case of a sigmoidal neural net $\mathcal{N}$ with piecewise rational activation functions, the upper bound $O\left(\ell^{2}\right)$ for the VC-dimension of $\tilde{\mathcal{N}}$ from Goldberg and Jerrum (1995) 
yields $\ell=\Omega\left(|\tilde{\mathcal{C}}|^{1 / 2}\right)$. In the case of a sigmoidal neural net with the sigmoid activation functions $\sigma(y)=1 /\left(1+\mathrm{e}^{-y}\right)$ (or other activation functions involving exponentiation) the upper bound $O\left(\ell^{4}\right)$ for the VC-dimension of $\tilde{\mathcal{N}}$ from Karpinski and Macintyre (1997) yields $\ell=\Omega\left(|\tilde{\mathcal{C}}|^{1 / 4}\right)$.

In the case of a first-order threshold circuit $\mathcal{N}$ one can show that $\ell \geqslant|\tilde{\mathcal{C}}| / \log (|\tilde{\mathcal{C}}|+1)$ by using a more direct counting argument from the proof of theorem 1(a) in Maass (1997c). An almost equivalent bound $\ell \geqslant|\tilde{\mathcal{C}}| /(1+\log |\tilde{\mathcal{C}}|)$ follows from the estimates in Cover (1968). Note that one cannot use here the upper bound from Baum and Haussler (1989) for the VC-dimension of a threshold circuit because that bound assumes that, instead of our $\ell$ chosen biases, all weights and biases of the threshold circuit are programmable parameters.

We will now discuss applications of theorem 3.1 to concrete classes $\mathcal{C}$ of subsets of $\left\{u_{1}, \ldots, u_{n}\right\}$, for which the computation of the associated boolean function $F_{\mathcal{C}}$ : $\{0,1\}^{n+|\mathcal{C}|} \rightarrow\{0,1\}$ appears to be of interest in the context of biological neural systems.

Application 1. Kreiter and Singer (1996) have shown that the firing of pairs of neurons in the motion-sensitive area MT of the visual cortex is synchronized when their individual receptive fields are crossed by a single moving bar. In contrast, if the same cells are activated by two different moving bars, responses show no or far fewer synchronous epochs.

Assume that a neuron in a higher cortical area wants to extract from these neurons in area MT the information whether a single moving object crosses at least $k$ vertically adjacent receptive fields, where $k$ is some given parameter. On the basis of the neural coding suggested by the results of Kreiter and Singer (1996) this neuron must compute the function $F_{\mathcal{C}}$ for $\mathcal{C}:=\left\{S \subseteq\left\{u_{1}, \ldots, u_{n}\right\}\right.$ : the neurons $u_{i} \in$ $S$ represent at least $k$ vertically adjacent locations\} for some fixed spatial map of the neurons $u_{1}, \ldots, u_{n}$. For simplicity we assume that the neurons $u_{1}, \ldots, u_{n}$ represent some $\ell \times m$ grid under their spatial map.

If $k \leqslant \ell / 2$, this class $\mathcal{C}$ contains a subclass $\tilde{\mathcal{C}}$ of $\geqslant n / 2$ sets with no set contained in another set. Hence a network of $\Omega(n / \log n)$ standard threshold gates or $\Omega\left(n^{1 / 2}\right)$ standard sigmoidal neurons with piecewise rational activation functions would be needed to compute this function $F_{\mathcal{C}}$. On the other hand, a single complex threshold gate can compute the function $F_{\mathcal{C}}$.

Application 2. Closely related to the previously discussed special cases is the so-called 'binding problem' (see e.g. Milner 1974, von der Malsburg 1981, Singer 1995, Shastri and Ajjanagadde 1993, Phillips and Singer 1997). Assume that objects in the outside world are analysed by sensory systems in terms of $m$ basic properties $P_{1}, \ldots, P_{m}$ from different categories (such as blue, round, large, approaching, honking, ... ). Furthermore assume that a collection $\mathcal{C}$ of subsets $H$ of $\left\{P_{1}, \ldots, P_{m}\right\}$ has been singled out because the existence of an object in the outside world that satisfies a combination $\bigwedge_{P_{j} \in H} P_{j}$ of basic properties in $H$ (e.g. large $\wedge$ approaching $\wedge$ honking) is of particular relevance for the system. On the other hand, it may be of no relevance for the systems if the same properties $P_{j} \in H$ are satisfied by different objects in the outside world (for example, if the human sensory systems detect in a busy street scene simultaneously a large house, an approaching child, and a honking car in a distant traffic jam).

It has been conjectured that neurons $v_{1}, \ldots, v_{m}$, where the firing rate $o\left(v_{i}\right)$ of neuron $v_{i}$ indicates the absence or presence of an object with basic property $P_{i}$, communicate through their firing correlation which basic properties are shared by the same object. Obviously 
a single complex threshold gate can then decide whether some combination $\bigwedge_{P_{j} \in H} P_{j}$ of basic properties is satisfied by a single object, since this amounts to computing the function $F_{\mathcal{C}}$ for the previously described class $\mathcal{C}$. On the other hand, according to theorem 3.1 neural networks of substantial size are needed to compute the same function $F_{\mathcal{C}}$ with standard sigmoidal neural nets.

The same example will be analysed further at the beginning of section 4 in the context of multi-layer computations.

Application 3. Many biological neural systems can select particular parts of their input for processing by higher layers, whereas the remainder of their input is ignored for the moment. This mechanism is referred to as 'attention' or 'awareness' and we refer to Koch and Crick (1994) for a detailed discussion of this problem in the context of neurophysiology.

One possible mechanism that has been put forward as a possible neurophysiological correlate of attention is a transient firing correlation of those sensory neurons whose receptive fields fall into the current region of attention. In this context a firing correlation would occur even among neurons that encode a static stimulus. Hence it has been conjectured that firing correlation in the context of attention is induced by internal mechanisms of the network, rather then by the stimulus itself.

Within the mathematical framework considered in this article one can model the use of firing correlation for mediating attention in the following way. We assume that there are $n$ input neurons $u_{1}, \ldots, u_{n}$, whose firing rates $o\left(u_{1}\right), \ldots, o\left(u_{n}\right)$ constitute the network input. In addition we assume that an additional neuron $u_{0}$ fires at a constant rate, and that the firing times of $u_{0}$ are correlated with the firing times of those input neurons $u_{i}$ which currently receive 'attention'. In other words, we assume that $c\left(\left\{u_{0}, u_{i}\right\}\right)$ is increased for those neurons $u_{i}$ that currently receive attention through some internal mechanism. This can take place without changing the firing rates $o\left(u_{i}\right)$ of these neurons. Under these assumptions a subsequent complex gate that computes $\sigma\left(\sum_{i=1}^{n} \alpha_{i} \cdot c\left(\left\{u_{0}, u_{i}\right\}\right) \cdot o\left(u_{0}\right) \cdot o\left(u_{i}\right)\right)$ can simulate any standard sigmoidal neuron that computes $\sigma\left(\sum_{i \in A} \alpha_{i} \cdot o\left(u_{i}\right)\right)$ for the set $A \subseteq\left\{u_{1}, \ldots, u_{n}\right\}$ of neurons that currently receive attention.

As a particularly simple special case consider the computational task to output 1 if and only if $o\left(u_{i}\right)=1$ for some $u_{i} \in A$. This amounts to the computation of the function $F_{\mathcal{C}}$ for the class $\mathcal{C}:=\left\{\left\{u_{0}, u_{i}\right\}: i=1, \ldots, n\right\}$, where $u_{0}$ is an additional input neuron with constant output value $o\left(u_{0}\right)=1$. According to theorem 3.1 a single complex threshold gate can carry out this task, whereas a neural network consisting of standard sigmoidal neurons needs to have a substantial number of gates to carry out the same computation. One noteworthy feature of the previously described computational task is that it can be solved by a single complex gate $v$ which has parameter values $\alpha_{v S} \neq 0$ only for sets $S$ of size 2 .

Larger separation results can easily be shown by theorem 3.1 for more complex computational tasks involving attention.

The last result of this section is not restricted to the new neural network models that are discussed in the other parts of this paper. It concerns the computational power of two different types of standard sigmoidal gates: high-order sigmoidal gates (also called $\Sigma \Pi$ units by Durbin and Rumelhart (1989)) and first-order sigmoidal gates. The 'order' of a sigmoidal gate is defined as the degree of the polynomial in the input variables that provides the argument for the acitivation function of the gate.

It is easy to exhibit a boolean function that can be computed by a single high-order threshold gate, but cannot be computed by a single first-order threshold gate. XOR of two bits is a well known example. It is substantially more difficult to exhibit a boolean function 
that can be computed by a single (or a few) higher-order gates, but requires a substantial number of first-order gates. The best previous result is that for PARITY of $n$ bits. This function can be computed by a single higher-order threshold gate (although it requires a gate of order $n$, and exponentially many polynomial terms if one encodes bits by 0,1 and not by $-1,1)$, whereas it requires $\Omega(\log n)$ gates in any feedforward circuit with first-order threshold gates (see chapter 7 in Siu et al 1995). Larger separation results have previously only been achieved with additional constraints on the structure of the first-order net, such as the constraint that its depth is extremely small and its weights are integers of polynomial size (Bruck and Smolenski 1992, Hajnal et al 1993, Siu et al 1995). Furthermore, these lower bounds can only be extended to analogue networks of first-order sigmoidal gates if one assumes that there is some fixed gap between analogue network outputs that correspond to 1 and those corresponding to 0 (see Maass et al 1994, Siu et al 1995).

The subsequent separation result holds for a boolean function that can be computed by a single second-order threshold gate (and a number of polynomial terms that is quadratic in the number $n$ of input bits). It is shown that substantially more than $\log n$ gates are required by any first-order neural network computing the same boolean function. Furthermore this lower bound holds for networks of first-order sigmoidal gates even without any extra condition regarding a gap between analogue network outputs corresponding to 1 versus those corresponding to 0 .

Corollary 3.2. A high-order sigmoidal gate has strictly more computational power than a first-order sigmoidal gate.

There exist for any $m \in \mathbf{N}$ boolean functions $F:\{0,1\}^{m} \rightarrow\{0,1\}$ that can be computed by a single high-order sigmoidal gate with $m / 2$ product terms of degree $\leqslant 2$, but where $F$ requires for its computation with first-order sigmoidal gates a multi-layer circuit with a substantial number of gates. The precise lower bounds depend on the activation function of the first-order sigmoidal gates: $F$ requires

- $\Omega(m / \log m)$ first-order threshold gates

- $\Omega\left(m^{1 / 2}\right)$ first-order sigmoidal gates with piecewise rational activation functions

- $\Omega\left(\mathrm{m}^{1 / 4}\right)$ first-order sigmoidal gates with activation functions that involve exponentiation.

We always assume that the analogue output of a sigmoidal neural net is rounded to yield a boolean output.

Proof. Choose $n \in \mathbf{N}$ maximal so that $2 n \leqslant m$. Set $\mathcal{C}:=\left\{S \subseteq\left\{u_{1}, \ldots, u_{n}\right\}:|S|=1\right\}$ in theorem 3.1. Then $F_{\mathcal{C}}$ is a function from $\{0,1\}^{2 n}$ into $\{0,1\}$, and $|\mathcal{C}|=\Omega(m)$. Obviously $F_{\mathcal{C}}$ can be computed by a single standard threshold gate with second-order terms.

Theorem 3.1 yields lower bounds for the size of arbitrary feedforward first-order sigmoidal neural nets that compute $F_{\mathcal{C}}$. These lower bounds are formulated in terms of $|\mathcal{C}|$, but since $|\mathcal{C}|=\Omega(m)$ they yield the same lower bounds in terms of $m$.

One can make $F_{\mathcal{C}}$ formally into a function $F$ from $\{0,1\}^{m}$ into $\{0,1\}$ by adding $m-2 n$ dummy input variables.

\section{Modelling multilayer computations with firing times and firing correlations}

In the preceding section we considered the case where the firing correlations $c(S)$ for sets $S \subseteq U$ of input neurons were given as part of the network input. In this section we consider the dual case where firing correlations among input neurons play no role, but internally generated firing correlations $c(T)$ for sets $T \subseteq\left\{v_{1}, \ldots, v_{m}\right\}$ of 'hidden' neurons 
become important for the computation. It turns out that the computational power of the network can be tremendously increased through the use of this new set of internal variables $c(T)$.

According to (2.2) the values of these correlation variables $c(T)$ are determined as follows:

$$
c(T)=\sigma_{c}\left(\sum_{u \in U} \alpha_{T u} \cdot o(u)+\sum_{S \subseteq U} \alpha_{T S} \cdot c(S) \cdot \prod_{u \in S} o(u)+\alpha_{T}\right) .
$$

In this equation $U$ is the set of all neurons which are the immediate predecessor of some neuron in the set $T$. The function $\sigma_{c}$ is some suitable activation function which scales the value of $c(T)$ into the desired range of this variable. For simplicity we assume in the following that $c(T)$ ranges over $[0,1]$, and that $\sigma_{c}$ is either the Heaviside function or some sigmoidal function (like the activation function $\sigma$ in equation (2.1)).

In a biological interpretation the parameters $\alpha_{T u}$ and $\alpha_{T S}$ in equation (4.1) can be used to model details of the geometrical and biochemical structure of the neurons in $T$ and $U$. The first term $\sum_{u \in U} \alpha_{T u} \cdot o(u)$ in (4.1) reflects the fact that the firing correlation of the neurons in $T$ can be increased through common input. Hence the value of $\alpha_{T u}$ should be chosen positive if neuron $u$ has excitatory synapses to all neurons in $T$, with roughly the same transmission delays to the soma of neurons in $T$. In fact, one may expect that the value of $\alpha_{T u}$ is in many cases proportional to $\min \left\{\alpha_{v u}: v \in T\right\}$.

The second term $\sum_{S \subseteq U} \alpha_{T S} \cdot c(S) \cdot \prod_{u \in S} o(u)$ in (4.1) reflects an alternative way in which firing correlation among neurons in $T$ can be achieved: if each neuron in $T$ receives input from some neuron $u \in S$ where $S$ is a set of presynaptic neurons that fire with a fairly large firing correlation (i.e. $c(S) \cdot \prod_{u \in S} o(u)$ is large). The parameter $\alpha_{T S}$ depends on the connectivity structure between the neuron sets $S$ and $T$, and on biochemical details of their synapses and of the dendritic trees of the neurons in $T$.

The computational model that is defined by the equations (2.1) and (4.1) makes it very easy to solve computational problems related to the so-called 'binding problem' for scenes with different objects (see our discussion in application 2 at the end of section 3). Assume again that there exists a set of $m$ basic properties $P_{1}, \ldots, P_{m}$ of objects, and a collection $\mathcal{C}$ of subsets $H$ of $\left\{P_{1}, \ldots, P_{m}\right\}$. Furthermore assume that for $H \in \mathcal{C}$ the combination $\bigwedge H:=\bigwedge_{P_{j} \in H} P_{j}$ of basic properties $P_{j}$ is of particular relevance for the neural system if it occurs in a single object. Finally assume that the computational goal of this system is to output 1 if the input indicates the presence of objects $O_{i}$ and $O_{\tilde{i}}$ so that $O_{i}$ satisfies $\wedge H$ and $O_{\tilde{i}}$ satisfies $\bigwedge \tilde{H}$, where $H$ and $\tilde{H}$ are two arbitrary but different sets in $\mathcal{C}$.

This concrete computational problem involves the 'binding problem', since it is required that one object $O_{i}$ satisfies all properties from some combination $H$ in $\mathcal{C}$, and one object $O_{\tilde{i}}$ satisfies all properties from some combination $\tilde{H}$ in $\mathcal{C}$. It would not suffice if both $O_{i}$ and $O_{\tilde{i}}$ each satisfy some properties in $H \cup \tilde{H}$, even if altogether all properties in $H \cup \tilde{H}$ get satisfied by at least one of the objects $O_{i}$ and $O_{\tilde{i}}$.

We will now construct a network $\mathcal{N}$ that solves this computational problem with computations that involve firing rates and firing correlations according to equations (2.1) and (4.1). We assume that the network $\mathcal{N}$ has $n$ input units $u_{1}, \ldots, u_{n}$, and that $o\left(u_{i}\right)=1$ indicates that object $O_{i}$ is present (else $\left.o\left(u_{i}\right)=0\right), i=1, \ldots, n$. On the second layer of the network $\mathcal{N}$ we have $m$ units $v_{1}, \ldots, v_{m}$ so that $o\left(v_{j}\right)=1$ if and only if there is some object $O_{i}$ present that has property $P_{j}$. We assume that the weights $\alpha_{j i}$ encode the knowledge about the basic properties $P_{j}$ of the objects $O_{i}: \alpha_{j i}=1$ if $O_{i}$ has property $P_{j}$, else $\alpha_{j i}=0$. If the output unit $v$ of the network receives information about the input only through the activations $o\left(v_{1}\right), \ldots o\left(v_{m}\right)$ of the units $v_{1}, \ldots, v_{m}$ on its first hidden layer, it cannot solve 


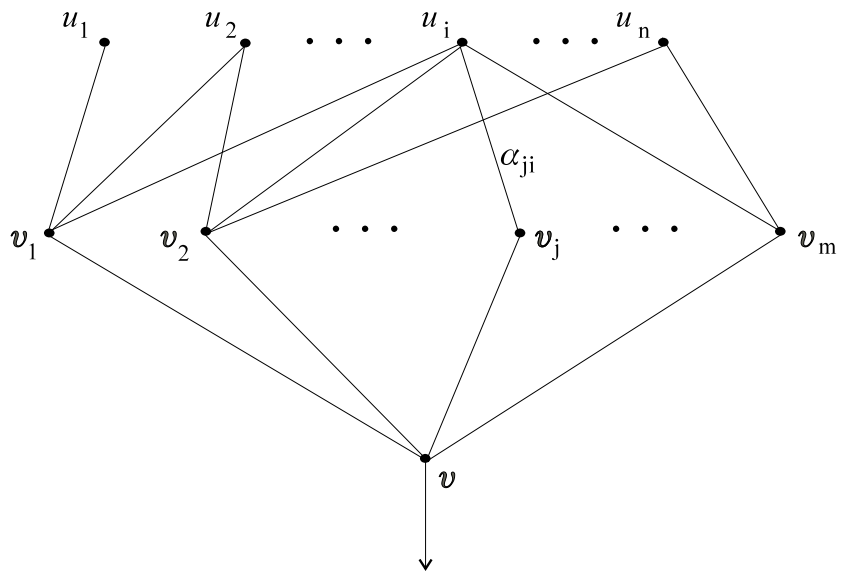

Figure 1. Construction of a network $\mathcal{N}$ of complex gates for the solution of a computational problem which is related to the 'binding problem'.

the computational problem that we consider. But if one takes into account that in addition the firing correlations $c(T)$ for subsets $T \subseteq\left\{v_{1}, \ldots, v_{m}\right\}$ can carry information about the input, then one can easily solve this problem.

This can be carried out by the following network $\mathcal{N}$ (see figure 1). We compute $c(T)$ according to equation (4.1) with $U=\left\{u_{1}, \ldots, u_{n}\right\}, \alpha_{T u_{i}}=\min \left\{\alpha_{j i}: v_{j} \in T\right\}$, $\alpha_{T S}=0$ for all $S$, and bias $\alpha_{T}=-1 / 2$. If one employs for simplicity the Heaviside activation function for $\sigma$ and $\sigma_{c}$, one has for $T_{H}:=\left\{v_{j}: P_{j} \in H\right\}$ that $c\left(T_{H}\right)=1$ if and only if $o\left(u_{i}\right)=1$ for some $i \in\{1, \ldots, n\}$ so that $\alpha_{j i}=1$ for all $P_{j} \in H$ (i.e. if an object $O_{i}$ is present that has property $P_{j}$ for all $\left.P_{j} \in H\right)$. Furthermore, one has $\prod_{P_{j} \in H} o\left(v_{j}\right)=1$ in this case.

We employ a complex gate $v$ as output gate on the third layer of $\mathcal{N}$. Its bias $\alpha_{v}$ receives the value 1.5 . Its parameter $\alpha_{v S}$ has value 1 if $S=\left\{v_{j}: P_{j} \in H\right\}$ for some set $H \in \mathcal{C}$, otherwise $\alpha_{v S}=0$. We set $\alpha_{v u}=0$ for all preceding neurons $u$.

According to (2.1) we have $o(v)=1$ if and only if $c\left(T_{H}\right) \cdot \prod_{P_{j} \in H} o\left(v_{j}\right)=1$ for at least two different sets $H$ in $\mathcal{C}$. Hence the network $\mathcal{N}$ outputs 1 if and only if its input $\left\langle o\left(u_{1}\right), \ldots, o\left(u_{n}\right)\right\rangle \in\{0,1\}^{n}$ satisfies $o\left(u_{i}\right)=1$ and $o\left(u_{\tilde{i}}\right)=1$ for some $i, \tilde{i} \in\{1, \ldots, n\}$ so that $O_{i}$ satisfies $\bigwedge H$ and $O_{\tilde{i}}$ satisfies $\bigwedge \tilde{H}$, where $H$ and $\tilde{H}$ are two arbitrary but different sets in $\mathcal{C}$. Therefore the network $\mathcal{N}$ solves the computational problem that we have considered.

One can easily see that a very similar network of complex gates can also handle the more realistic situation where the input units $u_{1}, \ldots, u_{n}$ represent $n$ 'sensors', and the presence of an object $O_{i}$ is signalled through correlated firing of a characteristic set $S_{i} \subseteq\left\{u_{1}, \ldots, u_{n}\right\}$ of these $n$ neurons. Formally, one has then $k$ subsets $S_{1}, \ldots, S_{k} \subseteq\left\{u_{1}, \ldots, u_{n}\right\}$, input variables $o\left(u_{1}\right), \ldots o\left(u_{n}\right)$ and $c\left(S_{i}\right)$ for $S_{i} \subseteq\left\{u_{1}, \ldots, u_{n}\right\}$, and one says that 'object $O_{i}$ is present' for some $i \in\{1, \ldots, k\}$ if $c\left(S_{i}\right) \cdot \prod_{u_{j} \in S_{i}} o\left(u_{j}\right)=1$. The architecture of a network $\mathcal{N}$ of complex gates that solves the same computational problem as before for this 'distributed' representation of objects $O_{i}$ can be chosen exactly as in the previous case. In this case the information as to which object $O_{i}$ has which basic property $P_{j}$ is encoded in the parameters $\alpha_{v S}$ for $S \subseteq\left\{u_{1}, \ldots, u_{n}\right\}$ and $v \in\left\{v_{1}, \ldots, v_{m}\right\}$, rather than in the parameters $\alpha_{j i}$. Furthermore the computation of $c(T)$ for sets $T \subseteq\left\{v_{1}, \ldots, v_{m}\right\}$ of hidden neurons according to equation (4.1) depends now on a proper choice of the parameters $\alpha_{T S}$ for sets 
$S \subseteq\left\{u_{1}, \ldots, u_{n}\right\}$. It no longer depends on the parameters $\alpha_{T_{u_{i}}}$, which can be chosen to be 0 in this more complex case. The construction of the output neuron $v$ remains unchanged. The preceding construction provides an example for a computation that involves two different roles of firing correlation within one computation (binding of sensors $u_{i}$, and binding of features $P_{j}$ ), as it might occur for example for visual processing in different areas of the visual cortex.

Finally, we would like to point out that the computational goal of the preceding network $\mathcal{N}$ has been chosen to be simple in order to illustrate the basic mechanisms. More complex computations involving different objects can easily be carried out if one replaces the output neuron $v$ of $\mathcal{N}$ by a more sophisticated subsequent network.

The preceding construction indicates in an informal way the new expressive possibilities that arise if a network can internally use firing correlations for its computation, even if the network input and output are given in terms of firing rates. In the following rigorous result we also consider neural computations where network inputs and outputs are given exclusively in terms of firing rates. We prove in theorem 4.1 and corollary 4.2 by straightforward arguments within the framework of computational complexity theory that the computational power of a neural net of some given size becomes substantially larger if it can internally employ auxiliary variables $c(T)$ that model firing correlations. In fact, it turns out that, without constraining rules on possible assignments of values to the parameters of a complex gate, their computational power is extremely large.

Theorem 4.1. Most functions $F:\{0,1\}^{n} \rightarrow\{0,1\}$ require exponentially in $n$ many gates for their computation on a feedforward network of standard threshold gates.

On the other hand all functions $F:\{0,1\}^{n} \rightarrow\{0,1\}$ can be computed by a neural net $\mathcal{N}$ with a fixed architecture that consists of just $2 n+2$ complex threshold gates.

Proof. The first part of the claim is well known. It follows immediately from Muroga et al (1961), where it was shown that the weights and the bias of any threshold gate with $k$ binary inputs can be chosen to be integers of absolute value $2^{O(k \cdot \log k)}$. The sharpest bound known is $2^{\frac{1}{2}(k+1) \log (k+1)-k}$ (Schmitt 1994). This implies that feedforward networks with arbitrary architecture and arbitrary real weights and biases consisting of $n$ input nodes and $m$ standard threshold gates can compute at most $2^{\left(n m+\frac{1}{2} m^{2}\right) \frac{1}{2}(n+m) \cdot \log (n+m)}$ different boolean functions $F:\{0,1\}^{n} \rightarrow\{0,1\}$. The logarithm of this estimate has value $o\left(2^{n}\right)$ for $m:=2^{n / 3} / n$. Therefore the fraction of functions $F:\{0,1\}^{n} \rightarrow\{0,1\}$ that can be computed with $\leqslant 2^{n / 3} / n$ standard threshold gates goes to 0 for $n \rightarrow \infty$.

The proof of the second part of theorem 4.1 is provided by the following construction of a network $\mathcal{N}$ that computes $F$. We construct a three-layer feedforward network $\mathcal{N}$ which employs complex gates on layers 2 and 3 . The computation of $\mathcal{N}$ proceeds according to equations (2.1) and (4.1). The architecture of $\mathcal{N}$ and the parameters of the gates on its first two layers are independent of the function $F$. Only the parameters of its output gate depend on the given function $F:\{0,1\}^{n} \rightarrow\{0,1\}$.

The first layer of $\mathcal{N}$ consists of $n$ input gates, $u_{1}, \ldots, u_{n}$, that encode the network input $\boldsymbol{x}=\left\langle x_{1}, \ldots, x_{n}\right\rangle \in\{0,1\}^{n}$ through their firing rates (i.e. $o\left(u_{i}\right)=x_{i}$ for $\left.i=1, \ldots, n\right)$. In addition $\mathcal{N}$ contains a gate $u_{0}$ that outputs $o\left(u_{0}\right)=1$, independently of the network input $\boldsymbol{x}$.

The second layer of $\mathcal{N}$ consists of $2 n$ complex gates, $v_{1}, \ldots v_{2 n}$. The weight on the edge from $u_{0}$ to $v_{j}$ has value 2 for $j=1, \ldots, 2 n$. For $i \in\{1, \ldots, n\}$ the weight on the edge from $u_{i}$ to $v_{2 i-1}$ also has value 2 . The bias of $v_{2 i-1}$ is set equal to -3 . The weight on the edge from $u_{i}$ to $v_{2 i}$ has value -2 , and the bias of $v_{2 i}$ is set to -1 . The weights $\alpha_{j i}$ on edges from $u_{i}$ to $v_{j}$ for $j \notin\{2 i-1,2 i\}$ have value 0 . Then we have $o\left(u_{i}\right)=o\left(v_{2 i-1}\right)=1-o\left(v_{2 i}\right)$ 


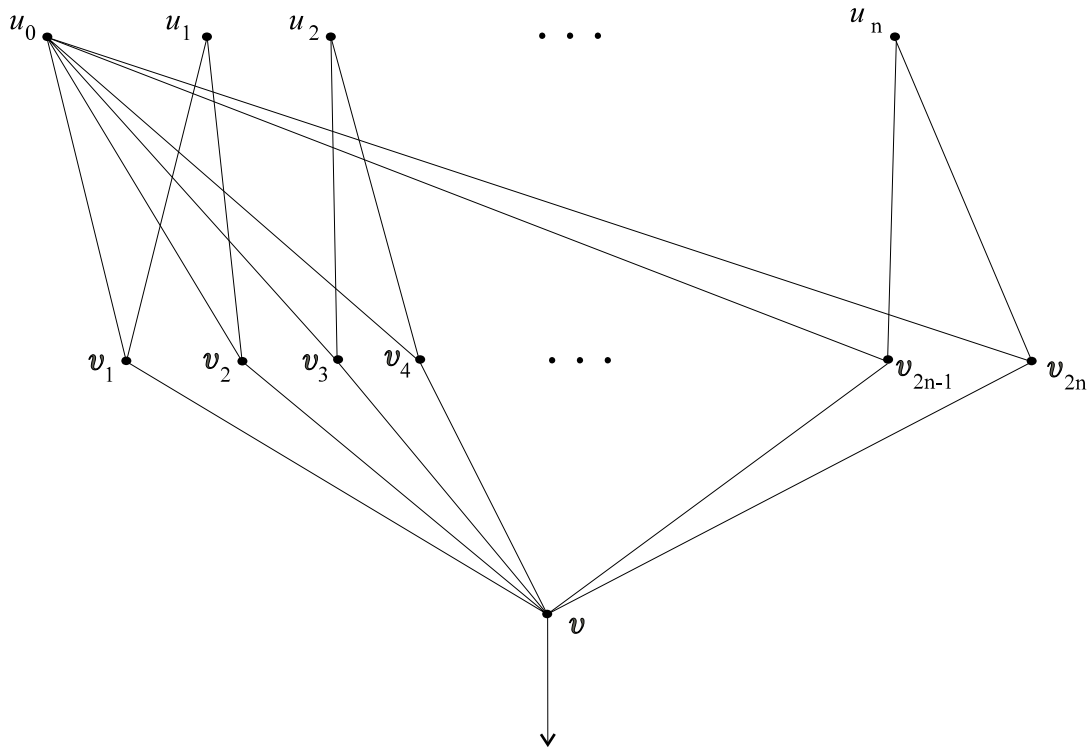

Figure 2. Construction of a small network $\mathcal{N}$ of complex gates for the proof of theorem 4.1.

for every network input $\boldsymbol{x} \in\{0,1\}^{n}$. Hence exactly $n$ gates on layer 2 output a 1 for every network input $\boldsymbol{x}$.

Since $u_{0}$ is the only neuron that has edges with non-zero weights to all $v_{j} \in T$, it is plausible to set $\alpha_{T u_{0}}=2$ and $\alpha_{T u_{i}}=0$ for $i \in\{1, \ldots, n\}$. With $\alpha_{T S}=0$ and $\alpha_{T}=-1$, one then has $c(T)=1$ for all subsets $T$ of $\left\{v_{1}, \ldots, v_{2 n}\right\}$ according to equation (4.1).

Hence for any network input $\boldsymbol{x} \in\{0,1\}^{n}$ one has $c(T)$. $\prod_{v_{j} \in T} o\left(v_{j}\right)=1$ for exactly one set $T$ of size $n$ (with $v_{2 i-1} \in T$ if $x_{i}=1$ and $v_{2 i} \in T$ if $x_{i}=0$ ). We will denote this set by $T_{x}$ in the following. Note that $c(T) \cdot \prod_{v_{j} \in T} o\left(v_{j}\right)=0$ for all sets $T$ of size $\geqslant n$ with $T \neq T_{x} \cdot \dagger$

The computation of the complex gate $v$ on layer 3 of $\mathcal{N}$ proceeds according to equation (2.1). Its bias is set to $-1 / 2$, and its weights on edges from preceding gates $v_{i}$ are all set to $0(i=1, \ldots, 2 n)$. Its weight $\alpha_{v S}$ for a set $S \subseteq\left\{v_{1}, \ldots, v_{2 n}\right\}$ is set to 1 if $S \supseteq T_{\boldsymbol{x}}$ for some $\boldsymbol{x} \in\{0,1\}^{n}$ with $F(\boldsymbol{x})=1$, otherwise $\alpha_{v S}=0$.

The output gate $v$ of this network $\mathcal{N}$ outputs $o(v)=F(\boldsymbol{x})$ for any network input $\boldsymbol{x} \in\{0,1\}^{n}$. Hence the network $\mathcal{N}$ consisting of $2 n+2$ gates (in addition to the input gates $u_{1}, \ldots, u_{n}$ ) computes the arbitrarily given function $F:\{0,1\}^{n} \rightarrow\{0,1\}$.

As an aside, we would like to point out that the parameters of $\mathcal{N}$ were chosen in such a way that for all network inputs the input of the Heaviside activation function of any gate in $\mathcal{N}$ is bounded away from zero. Therefore one can apply standard transformations to carry out the same computations also with smooth activation functions, such as the logistic sigmoid, instead of the Heaviside function.

$\dagger$ The present construction shows that according to our formalism one may have $c(T)=1$ (because of common excitatory input from neuron $u_{0}$ ), although several $v_{j} \in T$ are prevented from firing (i.e. $o\left(v_{j}\right)=0$ ) because of inhibitory input from other neurons. This shows that the values which our formalism assigns to "correlation variables' $c(S)$ may not have a meaningful interpretation if some neurons in $S$ do not fire (i.e. $o(u)=0$ for some $u \in S$ ). But fortunately the values of these variables $c(S)$ are irrelevant for all computations, because they are multiplied by zero in both equations (2.1) and (4.1). Note also that the value of the term (2.3) is undefined in this case, and hence does not single out a particular value of $c(S)$ as the appropriate one for this case. 
Corollary 4.2. If a function $F:\{0,1\}^{n} \rightarrow\{0,1\}$ can be defined by a boolean formula in disjunctive normal form with $k$ terms, then $F$ can be computed by a neural net $\mathcal{N}$ consisting of at most $2 n+2$ complex gates which have non-zero coefficients for just $k$ product terms.

Proof. For the output gate $v$ of the neural net $\mathcal{N}$ one sets $\alpha_{v S}=1$ if $S$ corresponds to a monomial in the disjunctive normal form of $F$; otherwise one sets $\alpha_{v s}=0$. The rest of the construction of theorem (4.1) remains unchanged.

Remark 4.3. Some recent experimental results (e.g. deCharms and Merzenich 1996, Vaadia et al 1995) show that some biological neural systems change their firing correlations in response to a stimulus, whereas the firing rates of the neurons involved do not change. This computational mode corresponds to the special case of our model where all firing rates in (2.1) and (4.1) (except for the firing rates of input and output neurons) are constants. It turns out that this special case of our model, which no longer contains any high-order terms if $c(S)=0$ for any set $S$ of input neurons, is computationally still very powerful.

For example, one can compute in this way the NP-complete decision problem CLIQUE on a linear-size feedforward circuit of complex threshold gates with one hidden layer. This is possible because the computation of this circuit can employ super-polynomically many correlation variables. We assume here that the absence or presence of each edge in the input graph $G$ with $n$ nodes is indicated by a separate binary variable, and that the output of CLIQUE is 1 if and only if $G$ has a clique of size $\geqslant n / 2$.

We assume that $c(S)=0$ for any set $S$ of input neurons, and that the input is given through the firing rates of the input neurons. We arrange that the firing rates $o(u)$ of all hidden neurons $u$ are constants. Therefore all product terms $\prod_{u \in S} o(u)$ for sets $S$ of hidden neurons that occur in the computation of this circuit can be replaced by constants. Hence all terms $c(S) \cdot \prod_{u \in S} o(u)$ for arbitrary sets $S$ of neurons on any layer of this network can be replaced by linear terms. In the hidden layer of the network we employ one complex gate $v$ for each node of the input graph $G$. For any set $T$ of $n / 2$ of these hidden units $v$ one arranges that $c(T)=1$ if and only if $T$ is a clique in $G$ (set $\alpha_{T u}=1$ for an input node $u$ if $u$ represents an edge whose incident vertices both belong to $T$; otherwise set $\alpha_{T u}=0$; the bias $\alpha_{T}$ is set to $-\left(\begin{array}{c}n / 2 \\ 2\end{array}\right)$ ).

\section{Conclusions}

We have introduced a simple framework for modelling neural computations with firing rates and firing correlations. In contrast to networks of spiking neurons, these new models need not keep track of individual firing times. Therefore they provide a suitable framework for a theoretical investigation of large-scale features of neural computations with firing rates and firing correlations. In contrast to previous models for modelling computational effects of firing correlations, our new models are general purpose computational models, whose function is not restricted to special types of computations.

We have presented several constructions of computationally powerful networks in this framework. Our constructions show that besides possible uses in solutions of the 'binding problem', firing correlations may also play other roles in the context of complex computations. Furthermore we present for the first time a proof that a neural network model that reflects salient aspects of neural computations with firing rates and firing correlations has strictly more computational power than a network of the same size that just models neural computations in terms of firing rates. 
Additionally, one of our separation results also has implications for standard neural network models. Corollary 3.2 provides the first proof that a high-order sigmoidal neural net can have strictly more computational power than any first-order sigmoidal neural net of a substantially larger size, without imposing constraints on the architecture, parameters or (reasonable) choice of activation functions employed by the first-order sigmoidal neural net.

We hope that the notion of a complex gate that is introduced in this paper provides a useful reference model for evaluating the computational capabilities of biological neurons. On the other hand, our model is sufficiently abstract to also provide a reference model for evaluating the computational capabilities of related computational units in pulsed VLSI. In both cases our model gives rise to interesting new questions regarding the possible values of parameters of a complex gate which can be realized in that setting. In fact, the model introduced in this paper may turn out to be a useful 'transmission tool' for moving computational mechanisms and principles from biological neurons to silicon implementations. In addition, it appears to be useful for quantifying specific advantages of silicon implementations of computations with correlated pulse trains; for example, new parameter ranges that can be achieved for the parameters of a complex gate in silicon but not in wetware.

Finally, we would like to point out that the new computational model that has been presented in this paper also has consequences for the investigation of learning in neural systems. The function that is computed by a network of complex gates depends on new parameters, and the question arises how these parameters can be tuned by suitable learning rules. It should be noted that according to theorem 4.1 a fixed network architecture of linear size with just one layer of 'adjustable' complex gates already provides a universal computational model (although only for binary inputs and outputs). This points to the possibility that instead of biologically dubious learning rules for multi-layer networks it may suffice to explore learning rules for tuning a larger set of parameters for a single layer of complex gates.

\section{Acknowledgments}

I would like to thank Thomas Natschläger and two anonymous referees for helpful comments.

\section{References}

Abeles M 1991 Corticonics (Cambridge: Cambridge University Press)

Baum E B and Haussler D 1989 What size net yields valid generalization? Neural Comput. 1 151-60

Bernander Ö, Koch C and Usher M 1994 The effect of synchronized inputs at the single neuron level Neural Comput. 6 622-41

Bower J M and Beeman D 1995 The Book of GENESIS: Exploring Realistic Neural Models with the GEneral NEural SImulation System (Berlin: Springer)

Bruck J and Smolenski R 1992 Polynomial threshold functions, AC ${ }^{0}$ functions, and spectral norms SIAM J. Comput. $2133-42$

Cover T M 1968 Capacity problems for linear machines Pattern Recognition ed L Kanal (Thompson Book Co) pp 283-9

deCharms R C and Merzenich M M 1996 Primary cortical representation of sounds by the coordination of actionpotential timing Nature 381 610-3 
Durbin R E and Rumelhart D E 1989 Product units: a computationally powerful and biologically plausible extension to backpropagation networks Neural Comput. 1 133-42

Eckhorn R, Reitboeck H J, Arndt M and Dicke P 1990 Feature linking via synchronization among distributed assemblies: simulations of results from cat visual cortex Neural Comput. 2 293-307

Eggermont J J 1990 The Correlative Brain: Theory and Experiment in Neural Interaction (Berlin: Springer)

Engel A K, Pönig K, Kreiter A K, Schillen T B and Singer W 1992 Temporal encoding in the visual cortex: new vistas on integration in the nervous system Trends Neurosci. 15 218-26

Gerstner W 1995 Time structure of the activity in neural network models Phys. Rev. E 51 738-58

Goldberg P W and Jerrum M R 1995 Bounding the Vapnik-Chervonenkis dimension of concept classes parameterized by real number Machine Learning 18 131-48

Hajnal A, Maass W, Pudlak P, Szegedy M and Turan G 1993 Threshold circuits of bounded depth J. Comput. System Sci. 46 129-54

Karpinski M and Macintyre A 1997 Polynomial bounds for VC-dimension of sigmoidal and general Pfaffian neural networks J. Comput. System Sci. 54 169-76

Koch C and Crick F 1994 Some further ideas regarding the neuronal basis of awareness Large-Scale Neuronal Theories of the Brain ed C Koch and J L Davis (Cambridge, MA: MIT Press) pp 93-110

Koch C and Poggio T 1992 Multiplying with synapses and neurons Single Neuron Computation (New York: Academic) pp 315-45

Koiran P 1996 VC-dimension in circuit complexity. Proc. 11th IEEE Conf. on Computational Complexity (Piscataway, NJ: IEEE) pp 81-5

Kreiter A K and Singer W 1996 Stimulus-dependent synchronization of neuronal responses in the visual cortex of the awake macaque monkey J. Neurosci. 16 2381-96

Krüger J (ed) 1991 Neuronal Cooperativity (Berlin: Springer)

Lumer E D and Huberman B A 1992 Binding hierarchies: a basis for dynamic perceptual grouping Neural Comput. 4 341-55

Maass W 1996 Lower bounds for the computational power of networks of spiking neurons Neural Comput. 8 1-40

1997a Fast sigmoidal networks via spiking neurons Neural Comput. 9 279-304

-1997b Bounds for the computational power and learning complexity of analog neural nets SIAM J. Comput. 26 708-32

- 1997c Networks of spiking neurons: the third generation of neural network models Neural Networks $\mathbf{1 0}$ 1659-71 (extended abstract, under a different title, appeared in Advances in Neural Information Processing Systems 9 (Cambridge, MA: MIT Press) pp 211-7)

Maass W, Schnitger G and Sontag E D 1994 A comparison of the computational power of sigmoid and boolean threshold circuits Theoretical Advances in Neural Computation and Learning ed V Roychowdhury, K-Y Siu and A Orlitsky (Dordrecht: Kluwer Academic) pp 127-51

MacLeod K and Laurent G 1996 Distinct mechanisms for synchronization and temporal patterning of odor-encoding neural assemblies Science 274 976-9

Mel B W 1994 Information processing in dendritic trees Neural Comput. 6 1031-85

1997 SEEMORE: Combining color, shape and texture histogramming in a neurally-inspired approach to visual object recognition Neural Comput. 9 777-804

Milner P M. 1974 A model for visual shape recognition Psychol. Rev. 81 521-35

Muroga S, Toda I and Takasu S 1961 Theory of majority decision elements J. Franklin Inst. 271 376-418

Murray A and Tarassenko L 1994 Analogue Neural VLSI: A Pulse Stream Approach (London: Chapman and Hall)

Omlin C W and Giles C L 1996 Constructing deterministic finite-state automata in recurrent neural networks $J$. Assoc. Comput. Mach. $\mathbf{4 3} 937-72$

Phillips W A and Singer W 1997 In search of common foundations for cortical computation Behav. Brain Sci. in press, see http://www-psych.stir.ac.uk/ dms/phillips.bbs.html

Ripley B D 1996 Pattern Recognition and Neural Networks (Cambridge: Cambridge University Press)

Schmitt M 1994 On the size of weights for McCulloch-Pitts neurons Proc. 6th Italian Workshop on Neural Networks ed E Caianello (Singapore: World Scientific) pp 241-6

Shastri L and Ajjanagadde V 1993 From simple associations to systematic reasoning: a connectionist representation of rules, variables and dynamic bindings using temporal synchrony Behav. Brain Sci. 16 417-94

Sillito A M, Jones H E, Gerstin G C and West D C 1994 Feature-linked synchronization of thalamic relay cell firing induced by feedback Nature 369 479-82

Singer W 1995 Synchronization of neuronal responses as a putative binding mechanism The Handbook of Brain Theory and Neural Networks ed M A Arbib (Cambridge, MA: MIT Press) pp 960-4

Siu K-Y, Roychowdhury V and Kailath T 1995 Discrete Neural Computation: A Theoretical Foundation (Englewood Cliffs, NJ: Prentice Hall) 
Tuckwell H C 1988 Introduction to Theoretical Neurobiology vols 1 and 2 (Cambridge: Cambridge University Press)

Vaadia E, Aertsen A and Nelken I 1995 Dynamics of neuronal interactions cannot be explained by neuronal transients Proc. R. Soc. (Lond.) B 261 407-10

van Schaik A, Fragnière E and Vittoz E 1997 A silicon model of amplitude modulation detection in the auditory brainstem Advances in Neural Information Processing Systems 9 (Cambridge, MA: MIT Press) pp 741-7

von der Malsburg C 1981 The Correlation Theory of Brain Function Internal Report 81-2, Department of Neurobiology, Max Planck Institute for Biophysical Chemistry, Göttingen

Weiss S M and Kulikowski C A 1991 Computer Systems that Learn (San Mateo, CA: Morgan Kaufmann)

Zaghloul M L, Meador J L and Newcomb R W (ed) 1994 Silicon Implementations of Pulse Coded Neural Networks (Dordrecht: Kluwer Academic) 